\title{
Inverse Uniqueness in Interior Transmission Problem and Its Eigenvalue Tunneling in Simple Domain
}

\author{
Lung-Hui Chen \\ Department of Mathematics, National Chung Cheng University, 168 University Road, Min-Hsiung, \\ Chia-Yi County 621, Taiwan \\ Correspondence should be addressed to Lung-Hui Chen; mr.lunghuichen@gmail.com
}

Received 7 October 2015; Accepted 6 December 2015

Academic Editor: Ivan Avramidi

Copyright (C) 2016 Lung-Hui Chen. This is an open access article distributed under the Creative Commons Attribution License, which permits unrestricted use, distribution, and reproduction in any medium, provided the original work is properly cited.

We study inverse uniqueness with a knowledge of spectral data of an interior transmission problem in a penetrable simple domain. We expand the solution in a series of one-dimensional problems in the far-fields. We define an ODE by restricting the PDE along a fixed scattered direction. Accordingly, we obtain a Sturm-Liouville problem for each scattered direction. There exists the correspondence between the ODE spectrum and the PDE spectrum. We deduce the inverse uniqueness on the index of refraction from the discussion on the uniqueness anglewise of the Strum-Liouville problem.

\section{Introduction}

In this paper, we study the inverse spectral problem in the following homogeneous interior transmission problem:

$$
\begin{aligned}
\Delta w+k^{2} n(x) w & =0 & & \text { in } D ; \\
\Delta v+k^{2} v & =0 & & \text { in } D ; \\
w & =v & & \text { on } \partial D ; \\
\frac{\partial w}{\partial v} & =\frac{\partial v}{\partial v} & & \text { on } \partial D,
\end{aligned}
$$

where $v$ is the unit outer normal; $D$ is a simple domain in $\mathbb{R}^{3}$ containing the origin with the Lipschitz boundary $\partial D ; n(x) \epsilon$ $\mathscr{C}^{2}\left(\mathbb{R}^{3}\right) ; n(x)>0$, for $x \in D ; n(x)=1$, for $x \notin D$. Equation (1) is called the homogeneous interior transmission eigenvalue problem. We say $k \in \mathbb{C}$ is an interior transmission eigenvalue of (1) if there is a nontrivial pair of solutions $(w, v)$ such that $w, v \in L^{2}(D), w-v \in H_{0}^{2}(D)$. The last two conditions in (1) are the Sommerfeld radiations condition to ensure the uniqueness on the scattered waves. We assume that $n(x) \neq 1$ near $\partial D$ from its interior, which minimizes the support of $D$.
To ensure the uniqueness of the scattered solution, we impose the Sommerfeld radiation condition:

$$
\begin{aligned}
& \lim _{|x| \rightarrow \infty}|x|\left(\frac{\partial w}{\partial|x|}-i k w\right)=0 ; \\
& \lim _{|x| \rightarrow \infty}|x|\left(\frac{\partial v}{\partial|x|}-i k v\right)=0 .
\end{aligned}
$$

Problem (1) occurs naturally when one considers the scattering of the plane waves by certain inhomogeneity defined by an index of refraction inside the domain $D$. The inverse problem is to determine the index of refraction by the measurement of the scattered waves in the farfields. The inverse scattering problem plays a role in various disciplines of science and technology such as sonar and radar, geophysical sciences, medical imaging, remote sensing, and nondestructive testing in instrument manufacturing. For the origin of interior transmission eigenvalue problem, we refer to Kirsch [1] and Colton and Monk [2]. For theoretical study and historic literature, we refer to [1,3-13]. To study the existence or location of the eigenvalues is a subject of high research interest $[1,2,5,6,8,11,14-18]$. Weyl's type of asymptotics for the interior transmission eigenvalues is expected, even though problem (1) is defined in noncompact $\mathbb{R}^{3}$. In that 
case, the distribution of the eigenvalues is directly connected to certain invariant characteristics on the scatterer. In this regard, we apply the methods from entire function theory [19-23] to study the distributional laws of the eigenvalues. We also refer to [24] for the reconstruction of the interior transmission eigenvalues and [25] for a numerical description on the distribution of the eigenvalues. It is remarkable that an example on the nonuniqueness of the index of refraction is constructed in [4, Section 6] for the class of radially symmetric indices of refraction with a jump discontinuity of $n^{\prime}(|x|)$. Finding the optimal regularity assumption on the index of refraction to attain the uniqueness or the nonuniqueness remains an open problem. The breakthrough is made from the point of view of inverse Sturm-Liouville theory [18] that inverse $\mathscr{L}^{2}$-uniqueness on the radially symmetric index of refraction is obtained if certain extra local information [18, Theorem 1] is provided.

For the nonsymmetrically stratified medium, there are not too many known results $[6,7,16,17]$. In this paper, we mainly follow the complex analysis methods $[3,14,18,26,27]$ to study the nonsymmetrical scatterers as a series of onedimensional problems along the rays scattering from the origin. The analysis along each ray possibly has multiple intersection points with $\partial D$, so we expect certain tunneling effect in a penetrable domain. In this paper, the new perspective is the asymptotic analysis inside and outside the perturbation. We give a global uniqueness on the index of refraction in simple domain by stating the following result.

Theorem 1. Let $n^{1}, n^{2}$ be two unknown indices of refraction as assumed in (1). If they have the same set of eigenvalues, then $n^{1} \equiv n^{2}$.

\section{Preliminaries}

We apply Rellich's expansion in scattering theory. Firstly we expand the solution $(v, w)$ of $(1)$ in two series of spherical harmonics by Rellich's lemma [8, page 32] in the far-fields:

$$
\begin{aligned}
& v(x ; k)=\sum_{l=0}^{\infty} \sum_{m=-l}^{m=l} a_{l, m} j_{l}(k r) Y_{l}^{m}(\widehat{x}) ; \\
& w(x ; k)=\frac{1}{r} \sum_{l=0}^{\infty} \sum_{m=-l}^{m=l} b_{l, m} y_{l}(r) Y_{l}^{m}(\widehat{x}),
\end{aligned}
$$

where $r:=|x|, 0 \leq r<\infty ; \hat{x}=(\theta, \varphi) \in \mathbb{S}^{2} ; j_{l}$ is the spherical Bessel function of first kind of order $l$. The summations converge uniformly and absolutely on suitable compact subsets in $|r| \geq R_{0}$, for some $R_{0} \gg 0$. We note that expansion (3) holds for the Helmholtz equation outside the simple domain [8, page 31, Lemma 2.11]. Particularly, the spherical harmonics

$$
\begin{array}{r}
Y_{l}^{m}(\theta, \varphi):=\sqrt{\frac{2 l+1}{4 \pi} \frac{(l-|m|) !}{(l+|m|) !}} P_{l}^{|m|}(\cos \theta) e^{i m \varphi}, \\
m=-l, \ldots, l ; l=0,1,2, \ldots,
\end{array}
$$

is a complete orthonormal system in $\mathscr{L}^{2}\left(\mathbb{S}^{2}\right)$. Here,

$$
P_{l}^{m}(t):=\left(1-t^{2}\right)^{m / 2} \frac{d^{m} P_{l}(t)}{d t^{m}}, \quad m=0,1, \ldots, l,
$$

where the Legendre polynomials $P_{l}, l=0,1, \ldots$, form a complete orthogonal system in $\mathscr{L}^{2}[-1,1]$. By the orthogonality of the spherical harmonics, the functions in the form

$$
\begin{aligned}
v_{l, m}(x ; k) & :=a_{l, m} j_{l}(k r) Y_{l}^{m}(\widehat{x}) ; \\
w_{l, m}(x ; k) & :=\frac{b_{l, m} y_{l}(r ; k)}{r} Y_{l}^{m}(\widehat{x})
\end{aligned}
$$

satisfy the first two equations in (1) independently [8, page 227] for $r \geq R_{0}$.

Now we look for $k \in \mathbb{C}, a_{l, m}$, and $b_{l, m}$, if any satisfies the following independent system for $l \in \mathbb{N}_{0}$ and $-l \leq m \leq l$ :

$$
\begin{gathered}
\left.a_{l, m} j_{l}(k r)\right|_{r=R_{0}}=\left.b_{l, m} \frac{y_{l}(r ; k)}{r}\right|_{r=R_{0}} ; \\
\left.a_{l, m}\left[j_{l}(k r)\right]^{\prime}\right|_{r=R_{0}}=\left.b_{l, m}\left[\frac{y_{l}(r ; k)}{r}\right]^{\prime}\right|_{r=R_{0}} .
\end{gathered}
$$

In terms of elementary linear algebra, the existence of the coefficients $a_{l, m}$ and $b_{l, m}$ is equivalent to finding the zeros of the following functional determinant:

$$
\begin{aligned}
& D_{l}\left(k ; R_{0}\right) \\
& :=\operatorname{det}\left(\begin{array}{c}
\left.j_{l}(k r)\right|_{r=R_{0}}-\left.\frac{y_{l}(r ; k)}{r}\right|_{r=R_{0}} \\
\left.\left\{j_{l}(k r)\right\}^{\prime}\right|_{r=R_{0}}-\left.\left\{\frac{y_{l}(r ; k)}{r}\right\}^{\prime}\right|_{r=R_{0}}
\end{array}\right) \\
& =-j_{l}\left(k R_{0}\right) \frac{y_{l}^{\prime}\left(R_{0} ; k\right)}{R_{0}}+j_{l}\left(k R_{0}\right) \frac{y_{l}\left(R_{0} ; k\right)}{R_{0}^{2}} \\
& \quad+k j_{l}^{\prime}\left(R_{0}\right) \frac{y_{l}\left(R_{0} ; k\right)}{R_{0}},
\end{aligned}
$$

in which the system is independent of $m$ and $\hat{x}$. The forward problem describes the distribution of the zeros of $D_{l}\left(k ; R_{0}\right)$, while the inverse problem specifies the index of refraction $n$ by the topology of the zero set. In $[14,18,26,27]$, we have discussed the methods to find the zeros of $D_{l}\left(k ; R_{0}\right)$.

Let $k \in \mathbb{C}$ be a possible eigenvalue of (7). Applying the analytic continuation of the Helmholtz equation and Rellich's lemma $[8$, page $32,33,222]$, the solutions parameterized by $k$ solve

$$
\begin{gathered}
w(x ; k) \equiv v(x ; k), \quad x \notin D ; \\
\frac{\partial w(x ; k)}{\partial \nu} \equiv \frac{\partial v(x ; k)}{\partial \nu}, \quad x \notin D,
\end{gathered}
$$

outside the simple domain $D$.

We note that representation (3) initially holds outside $|x| \geq R_{0}$, and the core of many inverse problems is to extend the solution into the perturbation. For our case, we want to 
extend representation (3) into $|x| \leq R_{0}$ for some possible set of $k$. Let $\widehat{x}_{1} \in \mathbb{S}^{2}$ be a given scattered direction satisfying the following geometric condition:

the line segment from $\left(R_{0}, \widehat{x}_{1}\right)$ to $\left(r_{1}, \widehat{x}_{1}\right)$,

$$
r_{1} \leq R_{0},
$$

lie outside $D$ with $\left(r_{1}, \hat{x}_{1}\right) \in \partial D$.

For $\hat{x}_{1}$, we extend each Fourier coefficient $y_{l}(r ; k)$ with $k \in$ $\mathbb{C}$ determined by system (7) for all $l$, toward the origin until it meets the boundary $\partial D$ at $\left(r_{1}, \widehat{x}_{1}\right)$. Along the given $\hat{x}_{1}$, we apply the differential operator $\Delta+k^{2} n$ with

$$
\Delta=\frac{1}{r^{2}} \frac{\partial}{\partial r} r^{2} \frac{\partial}{\partial r}+\frac{1}{r^{2} \sin \varphi} \frac{\partial}{\partial \varphi} \sin \varphi \frac{\partial}{\partial \varphi}+\frac{1}{r^{2} \sin ^{2} \varphi} \frac{\partial^{2}}{\partial \theta^{2}}
$$

to $\left\{w_{l, m}(x)\right\}$, which accordingly can solve problem (1) replaced with the manmade radially symmetric index of refraction $n(x)=n(r \hat{x})=n\left(r \hat{x}_{1}\right)$ for all $\hat{x} \in \mathbb{S}^{2}$. More importantly, the interior transmission condition implies the following ODE:

$$
\begin{aligned}
y_{l}^{\prime \prime}(r ; k)+\left(k^{2} n\left(r \widehat{x}_{1}\right)-\frac{l(l+1)}{r^{2}}\right) y_{l}(r ; k) & =0 ; \\
D_{l}\left(k ; r_{1}\right) & =0 .
\end{aligned}
$$

If there is merely one intersection point for $\left[0, R_{0}\right] \times \widehat{x}_{1}$ with $\partial D$, then we set the initial conditions of $y_{l}(r ; k)$ according to the following condition:

$$
\lim _{r \rightarrow 0}\left\{\frac{y_{l}(r ; k)}{r}-j_{l}(k r)\right\}=0 .
$$

The behavior of the Bessel function $j_{l}(k r)$ near $r=0$ is found in [28, page 437]. We refer initial condition (14) to [18]. That is,

$$
D_{l}(k ; 0)=0
$$

We observe that the uniqueness of the ODE (7) is valid up to the boundary $\partial D$ :

$$
\begin{aligned}
a_{l, m} j_{l}(k r) & =b_{l, m} \frac{y_{l}(r ; k)}{r} ; \\
a_{l, m}\left[j_{l}(k r)\right]^{\prime} & =b_{l, m}\left[\frac{y_{l}(r ; k)}{r}\right]^{\prime}, \quad r=r_{1}, \hat{x}_{1} \in \mathbb{S}^{2} .
\end{aligned}
$$

In particular, $D_{l}\left(k ; r_{1}\right)=D_{l}\left(k ; R_{0}\right)$ by the uniqueness of ODE (13) along the line segment $\left(R_{0}, \widehat{x}_{1}\right)$ to $\left(r_{1}, \widehat{x}_{1}\right)$.

For $l \geq 0$, we can take $a_{l, m}=b_{l, m}=1$ in (16). From the point of view of the Helmholtz equation,

$$
\begin{gathered}
b_{l, m} \frac{y_{l}(r ; k)}{r} Y_{l}^{m}(\widehat{x}), \\
\frac{y_{l}(r ; k)}{r} Y_{l}^{m}(\widehat{x}),
\end{gathered}
$$

both satisfy the Sommerfeld radiation condition whenever $y_{l}(r ; k)$ solves (13) and (14). By the uniqueness implied by the Sommerfeld radiation condition, we can choose that

$$
b_{l, m}=1 \text {. }
$$

Using a similar argument, we deduce that

$$
a_{l, m}=1 \text {. }
$$

In general, the solution $y_{l}(r ; k)$ depends on the scattered direction $\hat{x}$ whenever entering the perturbation, so we denote the extended solution of (13) as $\hat{y}_{l}(r ; k)$ and accordingly the functional determinant as $\widehat{D}_{l}\left(k ; r_{1}\right)$. Thus, (13) is relabeled as

$$
\begin{aligned}
\widehat{y}_{l}^{\prime \prime}(r ; k)+\left(k^{2} n(r \widehat{x})-\frac{l(l+1)}{r^{2}}\right) \widehat{y}_{l}(r ; k) & =0 ; \\
\widehat{D}_{l}(k ; 0) & =0, \\
\widehat{D}_{l}\left(k ; r_{1}\right) & =0 .
\end{aligned}
$$

The eigenvalues of (20) are discussed in [14, 26, 27] by the singular Sturm-Liouville theory in [29-31].

However, we are working on a simple domain $D$ in this paper. Hence, we modify the solution extension into $D$ in previous discussion. Instead of (16), we now ask for any $k \in \mathbb{C}$ that satisfies the following conditions:

$$
\begin{gathered}
\left.j_{l}(k r)\right|_{r=\widehat{r}}=\left.\frac{\widehat{y}_{l}(r ; k)}{r}\right|_{r=\widehat{r}} ; \\
{\left.\left[j_{l}(k r)\right]^{\prime}\right|_{r=\widehat{r}}=\left.\left[\frac{\widehat{y}_{l}(r ; k)}{r}\right]^{\prime}\right|_{r=\widehat{r}}, \quad \widehat{r} \in \widehat{R},}
\end{gathered}
$$

in which $\widehat{R}$ is the intersection set along the scattered angle $\widehat{x}$ defined by

$$
\widehat{R}:=\{\widehat{r} \mid(\widehat{r}, \widehat{x}) \in \partial D\}=\left\{\widehat{r}_{1}, \ldots, \widehat{r}_{\widehat{M}}\right\},
$$

and we will discuss the well-posedness of $\hat{y}_{l}(r ; k)$. For each fixed $\hat{x} \in \mathbb{S}^{2},(21)$ provides an initial condition at $\widehat{r} \in \widehat{R}$. Hence, the solution $\widehat{y}_{l}(r)$ of

$$
\widehat{y}_{l}^{\prime \prime}(r ; k)+\left(k^{2} n(r \widehat{x})-\frac{l(l+1)}{r^{2}}\right) \widehat{y}_{l}(r ; k)=0
$$

is constructed piecewise from infinity to the origin, at which

$$
\widehat{D}_{l}(k ; 0)=0 .
$$

We put it as a lemma.

Lemma 2. For $k \in \mathbb{C}$, there is unique solution that satisfies (21), (22), (23), and (24) for the fixed $\hat{x} \in \mathbb{S}^{2}$.

By the assumption of (1), we deduce that $\widehat{R}$ is a finite discrete set and $\widehat{r}_{1}<\widehat{r}_{2}<\cdots<\widehat{r}_{\widehat{M}}$ for each fixed $\widehat{x} \in \mathbb{S}^{2}$, in the case that $(\alpha, \widehat{x}),(\beta, \widehat{x})$, and $(\gamma, \widehat{x})$ are any three consecutive points along the incident direction $\hat{x}$. Whenever $(\beta, \hat{x})$ is a tangent point at the boundary, we disregard it and consider 


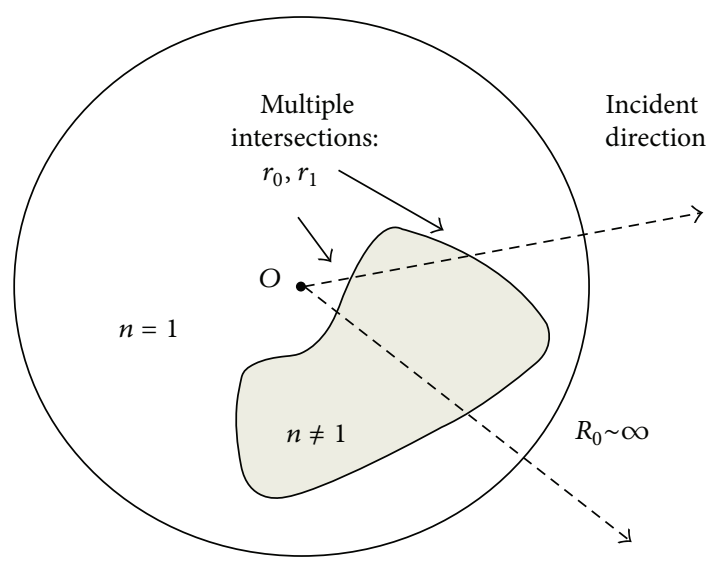

FIgURE 1: Rays of perturbation.

the line segment from $(\alpha, \widehat{x})$ to $(\gamma, \widehat{x})$ as either completely inside or outside the perturbation. Without loss of generality, we assume that $\widehat{R}$ contains no tangent point. See Figure 1.

To sketch an idea of how we construct a discrete set of $k$ for Lemma 2, we start with the first segment into the perturbation and discuss the well-posedness of the initial value problem starting at $\widehat{r}_{\widehat{M}}$ :

$$
\begin{aligned}
\hat{y}_{l}^{\prime \prime}(r)+\left(k^{2} n(r \widehat{x})-\frac{l(l+1)}{r^{2}}\right) \widehat{y}_{l}(r) & =0, \\
& \widehat{r}_{\widehat{M}-1} \leq r \leq \widehat{r}_{\widehat{M}} ; \\
\widehat{D}_{l}\left(k ; \widehat{r}_{\widehat{M}}\right) & =0,
\end{aligned}
$$

which has unique solution inward up to $\widehat{r}_{\widehat{M}-1}$ given that $j_{l}(k \widehat{r})$ is a known function for $k \in \mathbb{C}$. The behavior of the solution is understood by the singular Sturm-Liouville theory provided in Section 2. Because $\widehat{r}_{\widehat{M}}$ is the first intersection,

$$
w_{l, m}\left(\widehat{x} \widehat{r}_{\widehat{M}} ; k\right)=v_{l, m}\left(\widehat{x} \widehat{r}_{\widehat{M}} ; k\right),
$$

the uniqueness of (25) holds up to $\widehat{r}_{\widehat{M}}$ for $k \in \mathbb{C}$. We deduce from the unique analytic continuation that

$$
\begin{aligned}
w_{l, m}(x ; k) & =\frac{b_{l, m} y_{l}(r ; k)}{r} Y_{l}^{m}(\widehat{x}), \\
v_{l, m}(x ; k) & =a_{l, m} j_{l}(r ; k) Y_{l}^{m}(\widehat{x})
\end{aligned}
$$

hold outside $D$ for each fixed $k$. In particular, $\widehat{D}_{l}\left(k ; \widehat{r}_{\widehat{M}-1}\right)=$ 0 holds by the construction of $\hat{y}_{l}(r ; k)$. More importantly, the functional determinant $\widehat{D}_{l}\left(k ; \widehat{r}_{\widehat{M}-1}\right)=0$ is an algebraic condition that filters out a discrete set of eigenvalues from $\mathbb{C}$ for the problem

$$
\begin{aligned}
\widehat{y}_{l}^{\prime \prime}(r)+\left[k^{2} n(r \widehat{x})-\frac{l(l+1)}{r^{2}}\right] \widehat{y}_{l}(r) & =0, \\
& \widehat{r}_{\widehat{M}-1} \leq r \leq \widehat{r}_{\widehat{M}} ; \\
\widehat{D}_{l}\left(k ; \widehat{r}_{\widehat{M}}\right) & =0, \\
\widehat{D}_{l}\left(k ; \widehat{r}_{\widehat{M}-1}\right) & =0 .
\end{aligned}
$$

The spectral theory of (28) is simply singular Sturm-Liouville theory [29-31]. We have taken a similar approach in [14, $26,27,32]$. Let $k_{1}$ be one of its eigenvalues. Leaving the perturbation at $\widehat{r}_{\widehat{M}-1}$ toward the origin, $k_{1}$ defines another ODE system:

$$
\begin{aligned}
& \widehat{y}_{l}^{\prime \prime}(r)+\left[k_{1}^{2} n(r \widehat{x})-\frac{l(l+1)}{r^{2}}\right] \widehat{y}_{l}(r)=0, \\
& \widehat{r}_{\widehat{M}-2} \leq r \leq \widehat{r}_{\widehat{M}-1} ; \\
& \widehat{D}_{l}\left(k_{1} ; \widehat{r}_{\widehat{M}-1}\right)=0, \\
& \widehat{D}_{l}\left(k_{1} ; \widehat{r}_{\widehat{M}-2}\right)=0,
\end{aligned}
$$

in which $\widehat{D}_{l}\left(k_{1} ; \widehat{r}_{\widehat{M}-2}\right)=0$ holds due to the construction of $\hat{y}_{l}(r ; k)$ and the analytic continuation of the Helmholtz equation. The same $k_{1}$ appears at $\widehat{r}_{\widehat{M}-2}$ and is ready to define yet another new ODE:

$$
\begin{aligned}
& \hat{y}_{l}^{\prime \prime}(r)+\left[k_{1}^{2} n(r \widehat{x})-\frac{l(l+1)}{r^{2}}\right] \widehat{y}_{l}(r)=0, \\
& \widehat{r}_{\widehat{M}-3} \leq r \leq \widehat{r}_{\widehat{M}-2} ; \\
& \widehat{D}_{l}\left(k_{1} ; \widehat{r}_{\widehat{M}-2}\right)=0, \\
& \widehat{D}_{l}\left(k_{1} ; \widehat{r}_{\widehat{M}-3}\right)=0 .
\end{aligned}
$$

By the analytic continuation of

$$
w_{l, m}\left(x ; k_{1}\right)=\frac{b_{l, m} \hat{y}_{l}\left(r ; k_{1}\right)}{r} Y_{l}^{m}(\widehat{x})
$$

of the Helmholtz equation, the same $k_{1}$ satisfies (28), (29), and (30) and appears at $\widehat{r}_{\widehat{M}-3}$ and then consecutively into each intersection interval by its construction. That is, system (28) extends to $\mathbb{R}^{+}$.

There are several ways to produce an ODE flow from the origin to the infinity that satisfies

$$
\begin{gathered}
\widehat{D}_{l}\left(k ; \widehat{r}_{0}\right)=0, \\
\widehat{D}_{l}\left(k ; \widehat{r}_{1}\right)=0, \\
\vdots \\
\widehat{D}_{l}\left(k ; \widehat{r}_{\widehat{M}}\right)=0 .
\end{gathered}
$$

More importantly, system (30) inside $D$ also produces its group of eigenvalues that appear at $\widehat{r}_{\widehat{M}-3}$ and consecutively into all other intersection intervals by repeating the argument after (28). That is, system (30) extends to $\mathbb{R}^{+}$. In this way, we consider the piecewise construction of eigenfunctions for 
all possible discrete $k \in \mathbb{C}$ that make sense of the following system:

$$
\begin{gathered}
\widehat{y}_{l}^{\prime \prime}(r)+\left[k^{2} n(r \widehat{x})-\frac{l(l+1)}{r^{2}}\right] \widehat{y}_{l}(r)=0, \\
\quad \widehat{r}_{0}:=0<r<\infty ; \\
\widehat{D}_{l}\left(k ; \widehat{r}_{0}\right)=0, \\
\widehat{D}_{l}\left(k ; \widehat{r}_{1}\right)=0, \\
\vdots \\
\widehat{D}_{l}\left(k ; \widehat{r}_{\widehat{M}}\right)=0 .
\end{gathered}
$$

Each element of the zero set of $\widehat{D}_{l}\left(k ; \widehat{r}_{j}\right)$ defines an initial value condition for the ODE and an algebraic condition to filter out a discrete spectrum of (33). There is the uniqueness and the existence to the solution of (33) defined by the piecewise construction as shown above, and we call the extended solution $\hat{y}_{l}(r ; k)$ for each possible $k$ the eigenvalue tunneling in the interior transmission problem. In this paper, we use the solution $\hat{y}_{l}(r ; k)$ constructed as the procedure above. Such a construction can be set to initiate at $\widehat{r}_{0}$ and tunnels to the infinity.

We have already discussed the simple case in the radially symmetric and starlike domains [14, 26, 27]:

$$
\begin{aligned}
\widehat{y}_{l}^{\prime \prime}(r)+\left(k^{2} n(r \hat{x})-\frac{l(l+1)}{r^{2}}\right) \widehat{y}_{l}(r) & =0, \\
& \widehat{r}_{0}<r<\infty ; \\
\widehat{D}_{l}\left(k ; \widehat{r}_{0}\right) & =0, \\
\widehat{D}_{l}\left(k ; \widehat{r}_{1}\right) & =0 .
\end{aligned}
$$

For example, with the initial condition $\widehat{D}_{l}\left(k ; \widehat{r}_{0}\right)=0$, the function $\widehat{D}_{l}\left(k ; \widehat{r}_{1}\right)$ is an entire function of exponential type $[3,4,14,26,27,32]$. Thus, the eigenvalues of (34) form a discrete set in $\mathbb{C}$, accumulate into the eigenvalues of (33), and tunnel to the infinity.

Conversely, once we find an eigenvalue of (33) for some $l$ along some $\hat{x}$, it solves (7) by the uniqueness of ODE up to $|x|=R_{0}$ and then (10) by the analytic continuation of the Helmholtz equation. Whenever we collect all such eigenvalues from each incident $\hat{x} \in \mathbb{S}^{2}$, they constitute the interior transmission eigenvalues of (1). The geometric characteristics of the perturbation are connected by rays of ODE system to the far-fields.

\section{Asymptotic Expansions and Cartwright- Levinson Theory}

To study the functional determinants $\widehat{D}_{l}(k ; r)$, we collect the following asymptotic behaviors of $\widehat{y}_{l}(r ; k)$ and $\widehat{y}_{l}^{\prime}(r ; k)$. For each fixed $\hat{x}$, we apply the Liouville transformation $[7,8,29-$ 31, 33]:

$$
\widehat{z}_{l}(\widehat{\xi}):=[n(r \widehat{x})]^{1 / 4} \widehat{y}_{l}(r ; k),
$$

where

$$
\widehat{B}(r):=\widehat{\xi}(r)=\int_{0}^{r}[n(\rho \widehat{x})]^{1 / 2} d \rho, \quad 0 \leq r \leq \widehat{r}_{1} .
$$

Here we recall that $n$ is 1 outside $D$ :

$$
\widehat{z}_{l}^{\prime \prime}+\left[k^{2}-q(\widehat{\xi})-\frac{l(l+1)}{\widehat{\xi}^{2}}\right] \widehat{z}_{l}=0,
$$

in which

$$
\begin{aligned}
q(\widehat{\xi}):= & \frac{n^{\prime \prime}(r \widehat{x})}{4[n(r \widehat{x})]^{2}}-\frac{5}{16} \frac{\left[n^{\prime}(r \widehat{x})\right]^{2}}{[n(r \widehat{x})]^{3}}+\frac{l(l+1)}{r^{2} n(r \hat{x})} \\
& -\frac{l(l+1)}{\widehat{\xi}^{2}} .
\end{aligned}
$$

For simplicity of the notation, we drop all the superscripts about $\hat{x}$ whenever the context is clear.

Definition 3. Let $f(z)$ be an integral function of order $\rho$ and let $N(f, \alpha, \beta, r)$ denote the number of the zeros of $f(z)$ inside the angle $[\alpha, \beta]$ and $|z| \leq r$. One defines the density function as

$$
\begin{aligned}
\Delta_{f}(\alpha, \beta) & :=\lim _{r \rightarrow \infty} \frac{N(f, \alpha, \beta, r)}{r^{\rho}}, \\
\Delta_{f}(\beta) & :=\Delta_{f}\left(\alpha_{0}, \beta\right),
\end{aligned}
$$

with some fixed $\alpha_{0} \notin E$ such that $E$ is at most a countable set $[21-23,34]$.

Lemma 4. The functional determinant $\widehat{D}_{l}(k ; r)$ is of order one and of type $r+\widehat{B}(r), \widehat{r}_{0} \leq r \leq \widehat{r}_{1}$. In particular, one has the following density identity:

$$
\Delta_{\widehat{D}_{l}(k ; r)}(-\epsilon, \epsilon)=\frac{r+\widehat{B}(r)}{\pi} .
$$

Proof. We begin with (8):

$$
\begin{aligned}
& \widehat{D}_{l}(k ; r)=-j_{l}(k r) \frac{\widehat{y}_{l}^{\prime}(r ; k)}{r}+j_{l}(k r) \frac{\widehat{y}_{l}(r ; k)}{r^{2}} \\
& +k j_{l}^{\prime}(k r) \frac{\widehat{y}_{l}(r ; k)}{r}=\frac{k j_{l}^{\prime}(k r) \widehat{y}_{l}(r ; k)}{r}\{1 \\
& \left.-\frac{1}{k} \frac{j_{l}(k r)}{j_{l}^{\prime}(k r)} \frac{\widehat{y}_{l}^{\prime}(r ; k)}{\widehat{y}_{l}(r ; k)}+\frac{1}{k r} \frac{j_{l}(k r)}{j_{l}^{\prime}(k r)}\right\} \\
& =\frac{k j_{l}^{\prime}(k r) \widehat{y}_{l}(r ; k)}{r}\left\{\widehat{\alpha}_{l}(k)+O\left(\frac{1}{k}\right)\right\},
\end{aligned}
$$

in which

$$
\widehat{\alpha}_{l}(k):=1-\frac{1}{k} \frac{j_{l}(k r)}{j_{l}^{\prime}(k r)} \frac{\widehat{y}_{l}^{\prime}(r ; k)}{\hat{y}_{l}(r ; k)} .
$$


We have

$$
\frac{j_{l}(k r)}{j_{l}^{\prime}(k r)}=O(1)
$$

outside the zeros of $j_{l}^{\prime}(k r)$; similarly,

$$
\frac{\widehat{y}_{l}^{\prime}(r ; k)}{\widehat{y}_{l}(r ; k)}=O(k)
$$

outside the zeros of $\hat{y}_{l}(r ; k)$. The term $\widehat{\alpha}(k)$ is bounded and bounded away from zero outside the zeros of $j_{l}^{\prime}(k r)$ and $\widehat{y}_{l}(r ; k)$ on real axis. The behaviors of the $\widehat{z}_{l}^{\prime}(r ; k)$ and $\widehat{z}_{l}(r ; k)$ of (35) are well-known from the works $[3,8,14,26,27,32,33,35]$. In particular, the following asymptotics hold. For $\xi>0$ and $\mathfrak{R} k \geq 0$, there is a constant $C$ such that

$$
\begin{aligned}
& \left|u_{l}(\xi ; k)-\frac{\sin \{k \xi-l(\pi / 2)\}}{k^{l+1}}\right| \\
& \leq C|k|^{-(l+1)} \frac{\exp \{|\Im k| \xi\}}{|k \xi|} ; \\
& \left|u_{l}^{\prime}(\xi ; k)-\frac{\cos \{k \xi-l(\pi / 2)\}}{k^{l}}\right| \leq C|k|^{-l} \frac{\exp \{|\Im k| \xi\}}{|k \xi|}
\end{aligned}
$$

and that

$$
\begin{aligned}
& \left|z_{l}(\xi ; k)-u_{l}(\xi ; k)\right| \\
& \quad \leq C\left(\frac{\xi}{1+|k \xi|}\right)^{l+1} \exp \{|\Im k| \xi\} E(\xi, k) ; \\
& \left|z_{l}^{\prime}(\xi ; k)-u_{l}^{\prime}(\xi ; k)\right| \\
& \quad \leq C\left(\frac{\xi}{1+|k \xi|}\right)^{l} \exp \{|\Im k| \xi\} E(\xi, k),
\end{aligned}
$$

with the bounded error term

$$
E(\xi ; k)=\exp \left\{\int_{0}^{\xi} \frac{t q(t)}{1+|k t|} d t\right\}-1 \text {. }
$$

Consequently, we can compute Lindelöf's indicator function $[14,22,23,26,27,34]$ for $\widehat{y}_{l}(r ; k)$ and then $\widehat{D}_{l}(k ; r)$ for (41):

$$
\begin{aligned}
h_{\widehat{D}_{l}(k ; r)}(\theta) & =h_{j_{l}^{\prime}(k r)}(\theta)+h_{\widehat{y}_{l}(r ; k)}(\theta) \\
= & (r+\widehat{B}(r))|\sin \theta|, \\
& \quad \theta \in[0,2 \pi], \widehat{r}_{0} \leq r \leq \widehat{r}_{1},
\end{aligned}
$$

in which $\widehat{B}(r)=\int_{0}^{r}[n(\rho \widehat{x})]^{1 / 2} d \rho$, and we apply an inequality of Lindelöf's indicator function in [22, page 51].

In case that $\widehat{\alpha}_{l}(k) \equiv 0$, instead of (48), we have

$$
\begin{aligned}
h_{\widehat{D}_{l}(k ; r)}(\theta) & =h_{j_{l}(k r)}(\theta)+h_{\widehat{y}_{l}(r ; k)}(\theta) \\
= & (r+\widehat{B}(r))|\sin \theta|, \\
& \quad \theta \in[0,2 \pi], \widehat{r}_{0} \leq r \leq \widehat{r}_{1} .
\end{aligned}
$$

Referring Cartwright theory to [22, page 251], we conclude that $\widehat{D}_{l}(k ; r)$ is of Cartwright's class, and the lemma is thus proven.

Let us discuss the special case that $\widehat{\alpha}_{l}(k) \equiv 0$.

Lemma 5. The meromorphic function $\widehat{\alpha}_{l}(k) \equiv 0$ if and only if $n(r \widehat{x}) \equiv 1$ along the incident angle $\widehat{x}$.

Proof. We begin with

$$
\frac{\widehat{y}_{l}^{\prime}(r ; k)}{\widehat{y}_{l}(r ; k)} \equiv k \frac{j_{l}^{\prime}(k r)}{j_{l}(k r)}
$$

as a meromorphic function in $k$. Referring to [29-31, 33], $j_{l}^{\prime}(k r)$ has zeros asymptotically distributed near the zeros of $\cos (k r), j_{l}(k r)$ near the zero set of $\sin (k r)$. A similar property holds for $\hat{y}_{l}^{\prime}(r ; k)$ and $\hat{y}_{l}(r ; k)$. Hence, whenever $j_{l}^{\prime}(k r)$ has a zero, $\widehat{y}_{l}^{\prime}(r ; k)$ has a zero; $\widehat{y}_{l}(r ; k)$ has one whenever $j_{l}(k r)$ has one. The two perturbations, $j_{l}(k r)$ and $\widehat{y}_{l}(r ; k)$, have the same set of Neumann and Dirichlet eigenvalues. By the inverse spectral uniqueness of Sturm-Liouville problem [3, 33], we have $n \equiv 1$. The sufficient condition is obvious. This proves the lemma.

Thus, Lemma 4 merely describes the eigenvalue density of problem (34). To describe the density for (33), we may apply the translation invariant properties of interior transmission eigenvalues. Alternatively, we may consider the problem from the point of view of uniqueness theorem of ODE as in Section 1. In $\left[\widehat{r}_{1}, \widehat{r}_{2}\right]$, except the previous eigenvalues of (34), we consider the new eigenvalue density of the problem produced on the second interval; that is,

$$
\begin{aligned}
& \widehat{y}_{l}^{\prime \prime}(r ; k)+\left(k^{2} n(r \hat{x})-\frac{l(l+1)}{r^{2}}\right) \widehat{y}_{l}(r ; k)=0, \\
& \widehat{r}_{1}<r<\widehat{r}_{2} ; \\
& \widehat{D}_{l}\left(k ; \widehat{r}_{1}\right)=0, \\
& \widehat{D}_{l}\left(k ; \widehat{r}_{2}\right)=0 .
\end{aligned}
$$

The density is zero, because $\hat{y}_{l}(r ; k) / r$ and $j_{l}(r k)$ satisfy the same differential equation and initial condition at $\widehat{r}_{1}$ until $\widehat{r}_{2}$. Thus, there are only trivial eigenfunctions in $\left[\widehat{r}_{1}, \widehat{r}_{2}\right]$. One can prove inductively the density in each intersection interval, so we state the following theorem.

Theorem 6. Let $\widehat{N}(\alpha, \beta, R)$ denote the number of the eigenvalues of (33) inside the angle $[\alpha, \beta]$ and $|z| \leq R$ in $\mathbb{C}$. One defines the density function as

$$
\widehat{\Delta}(\alpha, \beta):=\lim _{R \rightarrow \infty} \frac{\widehat{N}(\alpha, \beta, R)}{R} .
$$

Then

$$
\widehat{\Delta}(-\epsilon, \epsilon)=\frac{\left|\widehat{r} \chi_{D}\right|+\int_{0}^{\infty} n^{1 / 2}(\rho \widehat{x}) \chi_{D} d \rho}{\pi},
$$

in which $\chi_{D}$ is 1 in $D$, zero otherwise, and $|\cdot|$ is the Lebesgue measure. 
Proof. This is only Lemma 4 and the discussion on (51).

Now we refresh the idea of the eigenvalue tunneling.

Proposition 7. If $k$ solves the following ODE for some $l \geq 0$,

$$
\begin{aligned}
& \widehat{y}_{l}^{\prime \prime}(r)+\left(k^{2} n(r \hat{x})-\frac{l(l+1)}{r^{2}}\right) \widehat{y}_{l}(r)=0, \\
& \widehat{r}_{0}:=0<r<\infty ; \\
& \widehat{D}_{l}\left(k ; \widehat{r}_{0}\right)=0 ; \\
& \widehat{D}_{l}\left(k ; \widehat{r}_{1}\right)=0,
\end{aligned}
$$

then $k$ is the eigenvalue to the following system for the same $l$ also:

$$
\begin{array}{cl}
\widehat{y}_{l}^{\prime \prime}(r)+\left(k^{2} n(r \hat{x})-\frac{l(l+1)}{r^{2}}\right) \widehat{y}_{l}(r)=0, & \\
& 0<r<\infty ; \\
& \widehat{D}_{l}\left(k ; \widehat{r}_{0}\right)=0, \\
& \widehat{D}_{l}\left(k ; \widehat{r}_{1}\right)=0, \\
\vdots & \\
\widehat{D}_{l}\left(k ; \widehat{r}_{\widehat{M}}\right)=0 .
\end{array}
$$

Proof. Let the eigenvalue $k$ solve the system of (54) for some $l \geq 0$, in which the first two equations there give an entire function in $k$ and the third condition implies that the eigenvalues $k$ of (54) form a discrete set in $\mathbb{C}[14,26,27]$. With $k$ given by (54), we continue ODE system (54) with the mixed boundary condition:

$$
\begin{aligned}
& \widehat{y}_{l}^{\prime \prime}(r)+\left(k^{2} n(r \hat{x})-\frac{l(l+1)}{r^{2}}\right) \widehat{y}_{l}(r)=0, \\
& \widehat{r}_{1}<r<\widehat{r}_{2} ; \\
& \widehat{D}_{l}\left(k ; \widehat{r}_{1}\right)=0 ; \\
& \widehat{D}_{l}\left(k ; \widehat{r}_{2}\right)=0 .
\end{aligned}
$$

The second equation above with $\widehat{D}_{l}\left(k ; \widehat{r}_{1}\right)=0$ actually gives a new initial value problem at $\widehat{r}_{1}$; that is,

$$
\begin{gathered}
\left.j_{l}(k r)\right|_{r=\widehat{r}_{1}}=\left.\frac{\widehat{y}_{l}(r ; k)}{r}\right|_{r=\widehat{r}_{1}} ; \\
{\left.\left[j_{l}(k r)\right]^{\prime}\right|_{r=\widehat{r}_{1}}=\left.\left[\frac{\widehat{y}_{l}(r ; k)}{r}\right]^{\prime}\right|_{r=\widehat{r}_{1}} .}
\end{gathered}
$$

Similarly, $\hat{y}_{l}(r ; k)$ initiates at $\hat{r}_{2}$ again and then tunnels consecutively into the infinity with the same $k$ according to our construction in Section 1. The solution $j_{l}(k r)$ is a known function throughout $\mathbb{R}^{3}$. Hence, the given $k$ satisfies

$$
\begin{gathered}
\widehat{D}_{l}\left(k ; \widehat{r}_{0}\right)=0, \\
\widehat{D}_{l}\left(k ; \widehat{r}_{1}\right)=0, \\
\vdots \\
\widehat{D}_{l}\left(k ; \widehat{r}_{\widehat{M}}\right)=0 .
\end{gathered}
$$

This proves the lemma.

Definition 8. One calls the eigenvalues of (55) that contributed by

$$
\begin{aligned}
\widehat{y}_{l}^{\prime \prime}(r)+\left(k^{2} n(r \hat{x})-\frac{l(l+1)}{r^{2}}\right) \widehat{y}_{l}(r) & =0, \\
& 0<r<\infty ; \\
\widehat{D}_{l}\left(k ; \widehat{r}_{j}\right) & =0 ; \\
\widehat{D}_{l}\left(k ; \widehat{r}_{j+1}\right) & =0
\end{aligned}
$$

the eigenvalues generated in interval $\left[\widehat{r}_{j}, \widehat{r}_{j+1}\right]$, where $\widehat{r}_{j}$, $\widehat{r}_{j+1} \in \widehat{R}$.

In general, we can take $\widehat{r}_{j}$ as the reference point by proceeding with the previous argument.

Proposition 9. If $k$ satisfies the following ODE for some $l \geq 0$ with a nontrivial solution,

$$
\begin{aligned}
\widehat{y}_{l}^{\prime \prime}(r)+\left(k^{2} n(r \hat{x})-\frac{l(l+1)}{r^{2}}\right) \widehat{y}_{l}(r) & =0, \\
& 0<r<\infty ; \\
\widehat{D}_{l}\left(k ; \widehat{r}_{j}\right) & =0 ; \\
\widehat{D}_{l}\left(k ; \widehat{r}_{j+1}\right) & =0,
\end{aligned}
$$

then $k$ is the eigenvalue to the following system:

$$
\hat{y}_{l}^{\prime \prime}(r)+\left(k^{2} n(r \hat{x})-\frac{l(l+1)}{r^{2}}\right) \widehat{y}_{l}(r)=0,
$$

$0<r<\infty$;

$$
\begin{gathered}
\widehat{D}_{l}\left(k ; \widehat{r}_{0}\right)=0, \\
\vdots \\
\widehat{D}_{l}\left(k ; \widehat{r}_{j}\right)=0, \\
\widehat{D}_{l}\left(k ; \widehat{r}_{j+1}\right)=0,
\end{gathered}
$$


Proof. ODE (60) has a discrete spectrum. For any $k$ in its spectrum we can extend the solution to the infinity and to the origin as we have argued in Lemma 2 or in Proposition 7.

\section{A Proof of Theorem 1}

Let $k$ be an eigenvalue of (54). Then it solves (7) at $|x|=$ $R_{0}$, and $w(x ; k)$ is defined outside $D$ through the analytic continuation of the Helmholtz equation. System (7) is radially symmetrically defined. Thus, with this same $k$, we can solve the ODE solution of (55) along all other directions in $\mathbb{S}^{2}$ by the piecewise construction in Lemma 2. Accordingly, the Fourier coefficients $\left\{\widehat{y}_{l}(r ; k)\right\}$ are defined for all angle $\widehat{x}$, and $w(x ; k)$ is then extended into $D$ with the given $k$. That is, the ODE spectrum along each scattered angle makes a part of the PDE spectrum. The spectrum of (54) is a subset of (1).

If $n^{j}, j=1,2$, are two indices of refraction with solution $\widehat{y}_{l}^{j}(r ; k)$ and determinant $\widehat{D}_{l}^{j}(k ; r)$ and given with identical set of interior transmission eigenvalues, then for all $\widehat{r}_{1}$ and all $l$ the following Sturm-Liouville problems in form of (54) hold with identical set of eigenvalues:

$$
\begin{aligned}
\partial_{r}^{2} \widehat{y}_{l}^{j}(r)+\left(k^{2} n^{j}(r \hat{x})-\frac{l(l+1)}{r^{2}}\right) \hat{y}_{l}^{j}(r)=0, & \\
0<r<\widehat{r}_{1} ; & \\
\widehat{D}_{l}^{j}(k ; 0)=0 ; & \\
\widehat{D}_{l}^{j}\left(k ; \widehat{r}_{1}\right)=0, \quad & \quad j=1,2 .
\end{aligned}
$$

This is one-dimensional inverse Sturm-Liouville problem [14, $26,27,29-31,33]$. The argument in $[14,26,27]$ shows that $n^{1}$ and $n^{2}$ have identical solutions $\hat{y}_{l}^{1}(r ; k)$ and $\hat{y}_{l}^{2}(r ; k)$, which we briefly explain as follows. Lemma 4 suggests $n^{1}$ and $n^{2}$ have zero density of

$$
\Delta_{\widehat{D}_{0}^{j}\left(k ; \widehat{r}_{1}\right)}(-\epsilon, \epsilon)=\frac{\widehat{r}_{1}+\widehat{B}^{j}\left(\widehat{r}_{1}\right)}{\pi}, \quad j=1,2 .
$$

On the other hand, the equation

$$
\widehat{y}_{0}^{1}\left(\widehat{r}_{1} ; k\right)-\widehat{y}_{0}^{2}\left(\widehat{r}_{1} ; k\right)=0
$$

has only density of $\widehat{B}^{1}\left(\widehat{r}_{1}\right) / \pi$ or $\widehat{B}^{2}\left(\widehat{r}_{1}\right) / \pi$, and then we deduce from the maximal zero set density from Cartwright theory [22, page 251] that

$$
\widehat{y}_{0}^{1}\left(\widehat{r}_{1} ; k\right)-\widehat{y}_{0}^{2}\left(\widehat{r}_{1} ; k\right) \equiv 0
$$

In particular, they have the same set of Dirichlet eigenvalues. Similar argument shows that

$$
\partial_{r} \widehat{y}_{0}^{1}\left(\widehat{r}_{1} ; k\right)-\partial_{r} \widehat{y}_{0}^{2}\left(\widehat{r}_{1} ; k\right) \equiv 0 .
$$

Particularly, they have the same set of Neumann eigenvalues. Hence, inverse uniqueness of the Bessel operator [30, Theorems 1.2 and 1.3] is applied to (62) and proves that $n^{1}(r \widehat{x}) \equiv$ $n^{2}(r \hat{x})$ in $\left[\widehat{r}_{0}, \widehat{r}_{1}\right]$ for all $\widehat{x}$. In this case, we have $n^{1}(r \widehat{x})=n^{2}(r \widehat{x})$ for $r \in\left[\widehat{r}_{0}, \widehat{r}_{1}\right]$ for all $\hat{x}$.

Now we see that $n^{1}(r \hat{x})=n^{2}(r \hat{x})$ trivially in $\left[\widehat{r}_{1}, \widehat{r}_{2}\right]$. We use a similar argument on the interval $\left[\widehat{r}_{0}, \widehat{r}_{3}\right]$ :

$$
\begin{aligned}
\partial_{r}^{2} \widehat{y}_{l}^{j}(r)+\left[k^{2} n^{j}(r \hat{x})-\frac{l(l+1)}{r^{2}}\right] \widehat{y}_{l}^{j}(r)=0, & \\
0<r<\widehat{r}_{1} ; & \\
\widehat{D}_{l}^{j}(k ; 0)=0 ; & \\
\widehat{D}_{l}^{j}\left(k ; \widehat{r}_{3}\right)=0, & \quad j=1,2 .
\end{aligned}
$$

If $n^{1}$ and $n^{2}$ have the same set of interior transmission eigenvalues, then they have the same spectrum for (67). From previous discussion, we conclude that $n^{1}(r \hat{x})=n^{2}(r \hat{x})$ for $r \in\left[\widehat{r}_{0}, \widehat{r}_{3}\right]$ for all $\widehat{x}$. The argument holds for all intersection points inductively. This proves Theorem 1 .

\section{Conflict of Interests}

The author declares that there is no conflict of interests regarding the publication of this paper.

\section{Acknowledgment}

The author wants to thank Professor Chao-Mei Tu at NTNU for proofreading an earlier version of this paper.

\section{References}

[1] A. Kirsch, "The denseness of the far field patterns for the transmission problem," IMA Journal of Applied Mathematics, vol. 37, no. 3, pp. 213-225, 1986.

[2] D. Colton and P. Monk, "The inverse scattering problem for time-harmonic acoustic waves in an inhomogeneous medium," The Quarterly Journal of Mechanics \& Applied Mathematics, vol. 41, no. 1, pp. 97-125, 1988.

[3] T. Aktosun, D. Gintides, and V. G. Papanicolaou, "The uniqueness in the inverse problem for transmission eigenvalues for the spherically symmetric variable-speed wave equation," Inverse Problems, vol. 27, Article ID 115004, 2011.

[4] T. Aktosun, D. Gintides, and V. G. Papanicolaou, "Reconstruction of the wave speed from transmission eigenvalues for the spherically symmetric variable-speed wave equation," Inverse Problems, vol. 29, no. 6, Article ID 065007, 2013.

[5] F. Cakoni, D. Colton, and H. Haddar, "The interior transmission eigenvalue problem for absorbing media," Inverse Problems, vol. 28, no. 4, Article ID 045005, 2012.

[6] F. Cakoni, D. Colton, and D. Gintides, "The interior transmission eigenvalue problem," SIAM Journal on Mathematical Analysis, vol. 42, no. 6, pp. 2912-2921, 2010.

[7] D. Colton, L. Päivärinta, and J. Sylvester, "The interior transmission problem," Inverse Problems and Imaging, vol. 1, no. 1, pp. 13-28, 2007.

[8] D. Colton and R. Kress, Inverse Acoustic and Electromagnetic Scattering Theory, vol. 93 of Applied Mathematical Sciences, Springer, New York, NY, USA, 3rd edition, 2013. 
[9] M. Hitrik, K. Krupchyk, P. Ola, and L. P. Päivärinta, "The interior transmission problem and bounds on transmission eigenvalues," Mathematical Research Letters, vol. 18, no. 2, pp. 279-293, 2011.

[10] M. Hitrik, K. Krupchyk, P. Ola, and L. P. Päivärinta, "Transmission eigenvalues for elliptic operators," The SIAM Journal on Mathematical Analysis, vol. 43, no. 6, pp. 2630-2639, 2011.

[11] A. Kirsch, "On the existence of transmission eigenvalues," Inverse Problems and Imaging, vol. 3, no. 2, pp. 155-172, 2009.

[12] H. Y. Liu, "Schiffer's conjecture, interior transmission eigenvalues and invisibility cloaking: singular problem vs. nonsingular problem," in Geometric Analysis and Integral Geometry, vol. 598 of Contemporary Mathematics, pp. 147-154, American Mathematical Society, Providence, RI, USA, 2013.

[13] B. P. Rynne and B. D. Sleeman, "The interior transmission problem and inverse scattering from inhomogeneous media," SIAM Journal on Mathematical Analysis, vol. 22, no. 6, pp. 1755$1762,1991$.

[14] L.-H. Chen, "An uniqueness result with some density theorems with interior transmission eigenvalues," Applicable Analysis, vol. 94, no. 8, pp. 1527-1544, 2015.

[15] E. Lakshtanov and B. Vainberg, "Bounds on positive interior transmission eigenvalues," Inverse Problems, vol. 28, no. 10, Article ID 105005, 2012.

[16] E. Lakshtanov and B. Vainberg, "Weyl type bound on positive interior transmission eigenvalues," Communications in Partial Differential Equations, vol. 39, no. 9, pp. 1729-1740, 2014.

[17] E. Lakshtanov and B. Vainberg, "Remarks on interior transmission eigenvalues, Weyl formula and branching billiards," Journal of Physics A: Mathematical and Theoretical, vol. 45, no. 12, pp. 125-202, 2012.

[18] J. R. McLaughlin and P. L. Polyakov, "On the uniqueness of a spherically symmetric speed of sound from transmission eigenvalues," Journal of Differential Equations, vol. 107, no. 2, pp. 351-382, 1994.

[19] M. L. Cartwright, "On the directions of Borel of functions which are regular and of finite order in an angle," Proceedings of the London Mathematical Society, Series 2, vol. 38, pp. 503-541, 1933.

[20] M. L. Cartwright, Integral Functions, Cambridge University Press, Cambridge, UK, 1956.

[21] P. Koosis, The Logarithmic Integral I, Cambridge University Press, New York, NY, USA, 1997.

[22] B. Ja. Levin, Distribution of Zeros of Entire Functions, Translations of Mathematical Mongraphs, American Mathematical Society, Providence, RI, USA, 1972.

[23] B. Ja. Levin, Lectures on Entire Functions, Translation of Mathematical Monographs, vol. 150, AMS, Providence, RI, USA, 1996.

[24] J. Sun, "Estimation of transmission eigenvalues and the index of refraction from Cauchy data," Inverse Problems, vol. 27, no. 1, Article ID 015009, 2011.

[25] F. Zeng, T. Turner, and J. Sun, "Some results on electromagnetic transmission eigenvalues," Mathematical Methods in the Applied Sciences, vol. 38, no. 1, pp. 155-163, 2015.

[26] L.-H. Chen, "A uniqueness theorem on the eigenvalues of spherically symmetric interior transmission problem in absorbing medium," Complex Variables and Elliptic Equations, vol. 60, no. 2, pp. 145-167, 2015.

[27] L.-H. Chen, "On the inverse spectral theory in a nonhomogeneous interior transmission problem," Complex Variables and Elliptic Equations, vol. 60, no. 5, pp. 707-731, 2015.
[28] M. Abramowitz and I. A. Stegun, Eds., Handbook of Mathematical Functions, Applied Mathematics Series 55, National Bureau of Standards, Washington, DC, USA, 1956.

[29] R. Carlson, "Inverse spectral theory for some singular SturmLiouville problems," Journal of Differential Equations, vol. 106, no. 1, pp. 121-140, 1993.

[30] R. Carlson, "A Borg-Levinson theorem for Bessel operators," Pacific Journal of Mathematics, vol. 177, no. 1, pp. 1-26, 1997.

[31] R. Carlson, "Inverse Sturm-Liouville problems with a singularity at zero," Inverse Problems, vol. 10, no. 4, pp. 851-864, 1994.

[32] D. Colton, Y.-J. Leung, and S. Meng, "Distribution of complex transmission eigenvalues for spherically stratified media," Inverse Problems, vol. 31, no. 3, Article ID 035006, 2015.

[33] J. Pöschel and E. Trubowitz, Inverse Spectral Theory, Academic Press, Orlando, Fla, USA, 1987.

[34] R. P. Boas, Entire Functions, Academic Press, New York, NY, USA, 1954.

[35] V. A. Marchenko, Sturm-Liouville Operators and Applications, Birkhaeuser, Basel, Switzerland, 1986. 


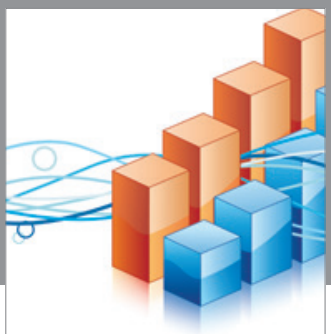

Advances in

Operations Research

vatem alat4

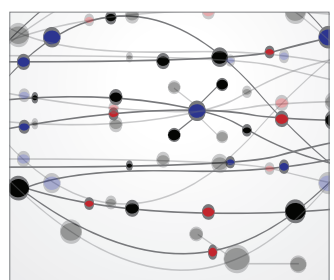

\section{The Scientific} World Journal
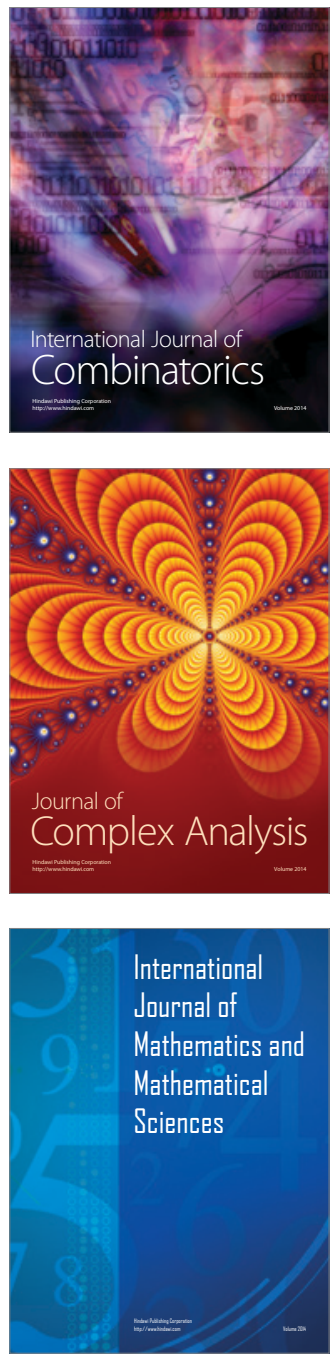
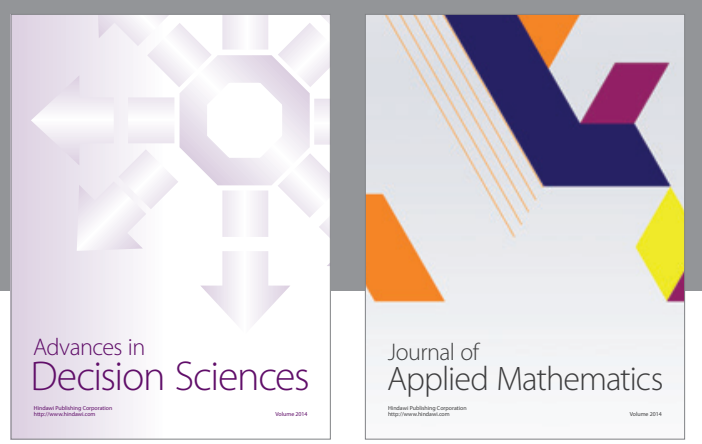

Algebra

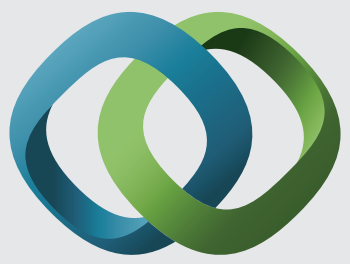

\section{Hindawi}

Submit your manuscripts at

http://www.hindawi.com
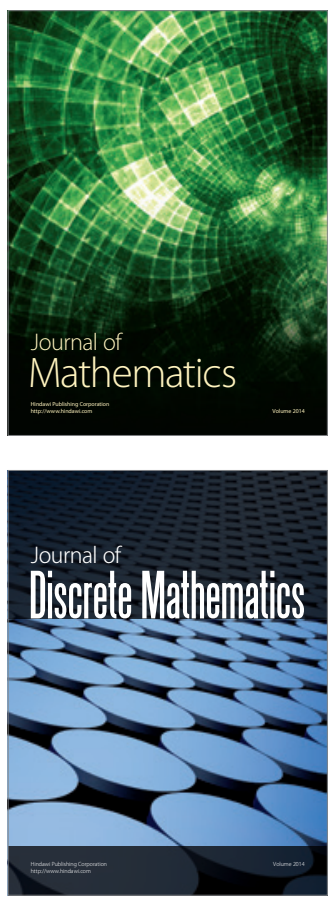

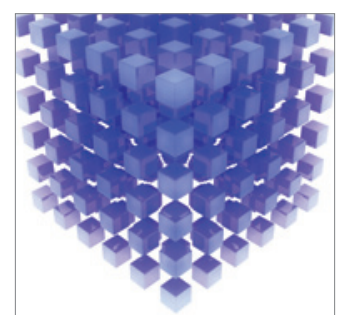

Mathematical Problems in Engineering
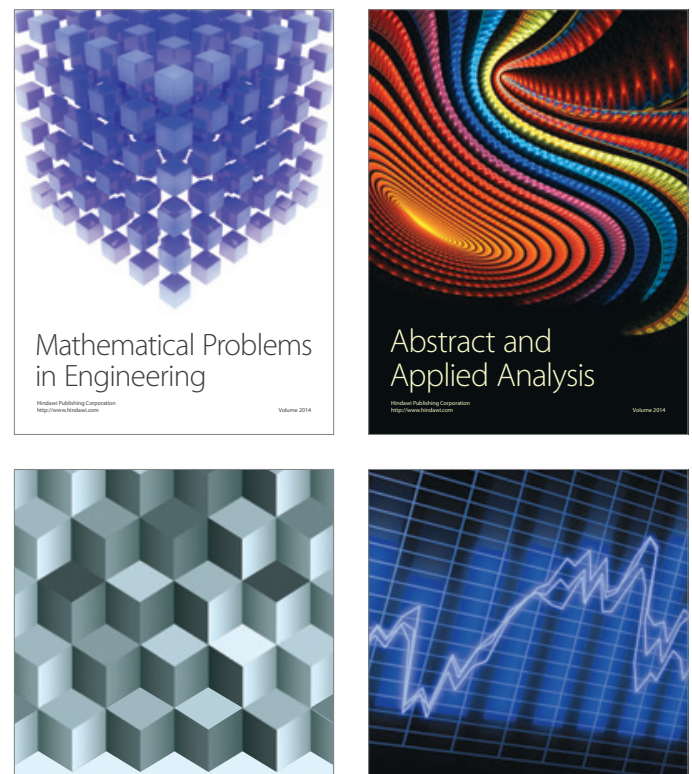

Journal of

Function Spaces

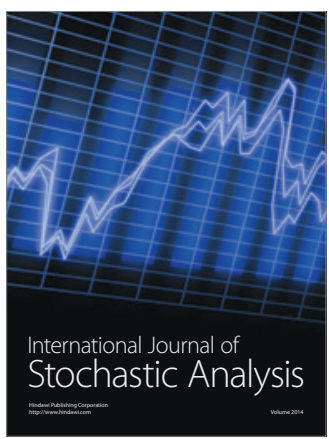

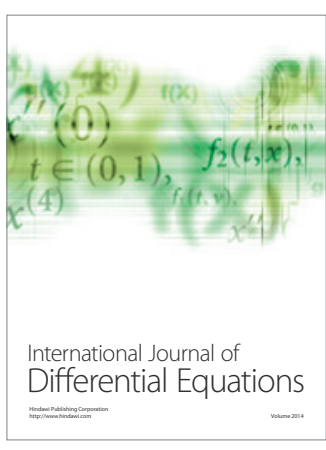
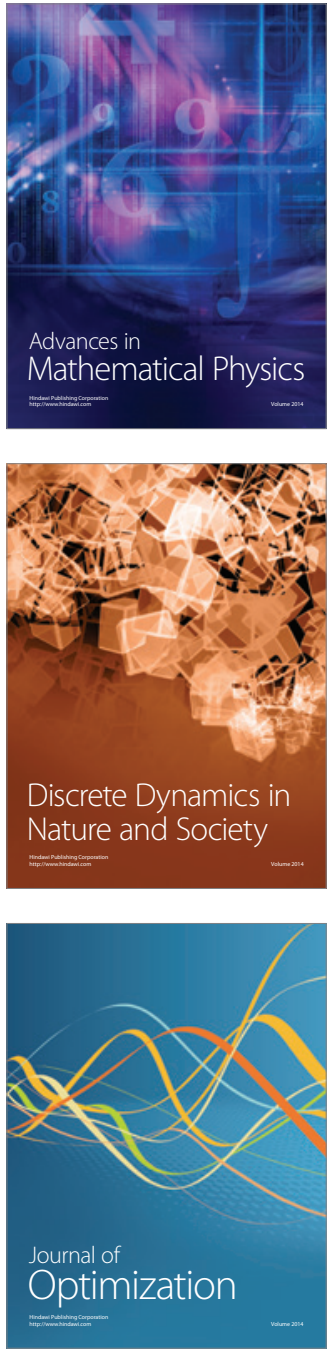